ESAIM: PROCEEDINGS, October 2011, Vol. 32, p. 211-230

E. Cancès, N. Crouseilles, H. Guillard, B. Nkonga, and E. Sonnendrücker, Editors

\title{
DISCONTINUOUS GALERKIN SEMI-LAGRANGIAN METHOD FOR VLASOV-POISSON
}

\author{
N. Crouseilles ${ }^{1}$, M. Mehrenberger ${ }^{2}$ And F. Vecil ${ }^{3}$
}

\begin{abstract}
We present a discontinuous Galerkin scheme for the numerical approximation of the onedimensional periodic Vlasov-Poisson equation. The scheme is based on a Galerkin-characteristics method in which the distribution function is projected onto a space of discontinuous functions. We present comparisons with a semi-Lagrangian method to emphasize the good behavior of this scheme when applied to Vlasov-Poisson test cases.

Résumé. Une méthode de Galerkin discontinu est proposée pour l'approximation numérique de l'équation de Vlasov-Poisson 1D. L'approche est basée sur une méthode Galerkin-caractéristiques où la fonction de distribution est projetée sur un espace de fonctions discontinues. En particulier, la méthode est comparée à une méthode semi-Lagrangienne pour l'approximation de l'équation de Vlasov-Poisson.
\end{abstract}

\section{INTRODUCTION}

The description of charged particles in a plasma can be done at the kinetic level using the simple onedimensional Vlasov-Poisson system. The solution $f(t, x, v)$ depends on the time $t \geq 0$, the space $x \in[0, L]$ and the velocity $v \in \mathbb{R}$. In dimensionless variables, the Vlasov-Poisson system reads

$$
\partial_{t} f+v \partial_{x} f+E \partial_{v} f=0, \quad \partial_{x} E=\int_{\mathbb{R}} f d v-1,
$$

with $E(t, x)$ the self-consistent electric field. The model is provided with an initial datum $f_{0}(x, v)$, periodic conditions in space and vanishing conditions in the velocity direction. Well-posedness of the problem is ensured under a mean electrostatic type condition: $\int_{0}^{L} E(t, x) d x=0$.

A lot of numerical methods have been developed for the approximation of (1). Particle methods [4], in which macro-particles solve the characteristics of the Vlasov equation, have been preferred for a long time because of their low computational cost. These methods are known to be noisy, which might prevent an accurate description of the distribution function, for example, in low density regions. On one side, the more recently developed Eulerian methods discretize the Vlasov equation on a mesh of the phase-space, thus improving the precision; among these Eulerian methods, many variants have been designed $[5,7,11]$. On the other side, discontinuous Galerkin (DG) approach has been introduced for the approximation of transport problems and presents the advantage of using very local data, even for high-order reconstruction. Therefore, coupling the advantages of the semi-Lagrangian method (no theoretical restriction on the time step) with a DG reconstruction seems an

\footnotetext{
1 INRIA, IRMA, Université de Strasbourg, 7, rue Ren Descartes, 67084 Strasbourg

2 IRMA, Université de Strasbourg, 7, rue Ren Descartes, 67084 Strasbourg

3 Departament de Matemàtica Aplicada, Universitat de València, calle Dr. Moliner 50, 46100 Burjassot (Spain)
}

(C) EDP Sciences, SMAI 2011 
attractive strategy. The main scope of this work is to explore and test the efficacy of this numerical method for the approximation of (1). In particular, numerical convergence towards analytical solutions and comparison to a reference method are carefully performed. Furthermore, comparisons with standard methods of the literature on classical plasma physics test cases are performed to evaluate the precision and the characteristics of the DG method.

As pointed out in first works on the subject, DG reconstruction $[1,2,12,13,19]$ presents interesting features; as mentioned above, it permits a local reconstruction even when high-order reconstruction is used, which is important when dealing with the parallelization for high-dimensional problems. For Vlasov-based systems, the method is attractive since it is inherently conservative and filamentation or strong gradients can be well described due to the easy use of high-order basis functions.

Thanks to a splitting procedure, the numerical resolution of the Vlasov equation can be reduced to a chain of linear advections and Poisson integrations. Therefore, the present study focuses on the numerical approximation of linear advection using a semi-Lagrangian scheme with a DG reconstruction. More precisely, by means of the Galerkin-characteristics formulation [3,15-17] the distribution function at the previous time is integrated on a Lagrangian cell. The projection space has been chosen to be the Lagrange polynomials interpolating the Gauß points in each cell (degrees of freedom). This results in an explicit scheme, because the mass matrix is block diagonal, thus allowing extensions to arbitrarily high orders. In particular, a stability analysis can be performed in the Fourier space [25].

The paper is organized as follows. In Section 1.1 the method is detailed for the one-dimensional linear advection case. Then, in Section 1.2 the stability is studied. Finally, in Section 2 numerical results are presented, with a specific attention to Vlasov-Poisson applications, for which comparisons with a semi-Lagrangian method which cubic splines reconstruction are performed.

\section{NumERICAL METHOD}

\subsection{D linear advection}

As precised in the introduction, we focus on solving the 1D linear advection problem

$$
\left\{\begin{array}{l}
\partial_{t} f+a \partial_{x} f=0, \\
f(t=0, x)=f^{0}(x),
\end{array}\right.
$$

where $f:[0,+\infty[\times \Omega \longrightarrow \mathbb{R}$ and $a$ is a real constant.

\subsubsection{Discretization}

DG space. - The domain $\Omega=[0,1]$ is partitioned into $N$ intervals $\left\{I_{i}=\left[x_{i-1 / 2}, x_{i+1 / 2}\right]\right\}_{i=0, \ldots, N-1}$, so that $\Omega=I_{0} \cup I_{1} \cup \ldots \cup I_{N-1}$. For the scope of this work, we shall take homogeneous intervals: $\Delta x=x_{1 / 2}-x_{-1 / 2}=$ $x_{3 / 2}-x_{1 / 2}=\ldots=x_{N-1 / 2}-x_{N-3 / 2}$. The projection space is denoted by $V^{d}$, which is a discontinuous finite element space

$$
V^{d}=\left\{\psi \in L^{2}(\Omega): \psi \in \mathbb{P}^{d}\left(I_{i}\right), \quad \text { for } i=0, \ldots, N-1\right\},
$$

where $\mathbb{P}^{d}\left(I_{i}\right)$ denotes the one-variable polynomials of degree at most $d$, on the interval $I_{i}$. Let define the standard projection from $L^{2}(\Omega)$ onto the finite element space $V^{d}$ :

$$
\omega \mapsto \omega_{i, j}=\left\langle\omega, \varphi_{i, j}\right\rangle=\int_{\Omega} \omega(x) \varphi_{i, j}(x) d x
$$

where the $\left\{\varphi_{i, j}\right\}_{(i, j) \in\{0, \ldots, N-1\} \times\{0, \ldots, d\}}$ are a basis for $V^{d}$.

The starting point of our strategy is the Galerkin-characteristics method (see [15, 16]). We introduce the 
following notation: $f^{n}(x) \simeq f\left(t^{n}, x\right)$ and the time step $\Delta t$ such that $t^{n}=n \Delta t$. The idea of integrating on an interval $I_{i}$ and following the characteristics backward, which, in the linear case, gives

$$
\begin{aligned}
\int_{x_{i-1 / 2}}^{x_{i+1 / 2}} f^{n+1}(x) \varphi(x) d x & =\int_{x_{i-1 / 2}}^{x_{i+1 / 2}} f^{n}\left(\mathcal{X}\left(t^{n} ; t^{n+1}, x\right)\right) \varphi(x) d x \\
& =\int_{x_{i-1 / 2}}^{x_{i+1 / 2}} f^{n}(x-a \Delta t) \varphi(x) d x \\
& =\int_{x_{i-1 / 2}-a \Delta t}^{x_{i+1 / 2}-a \Delta t} f^{n}(x) \varphi(x+a \Delta t) d x
\end{aligned}
$$

Here we have, first of all, written the solution at time $t^{n+1}$ in terms of the characteristics $\mathcal{X}(s ; t, x)$ defined by

$$
\left\{\begin{array}{l}
\frac{d}{d s} \mathcal{X}(s)=a \\
\mathcal{X}(t)=x
\end{array}\right.
$$

Then, we have written the explicit expression for the characteristics in the linear advection case, and finally we have changed variables.

Choice of the basis for $V^{d}$. - The intervals $I_{i}$ are subdivided into as many points as the order of the polynomials which we take into account, the subdivision being given by the Gauß points. In each interval $I_{i}$ we shall have $d+1$ points, denoted $\left\{x_{i, j}\right\}_{(i, j) \in\{0, \ldots, N-1\} \times\{0, \ldots, d\}}$. As a basis for $V^{d}$ we shall use the Lagrange polynomials interpolating on the Gauß points $x_{i, j}$, restricted to the interval $I_{i}$ :

$$
\varphi_{i, j}(x)=\left\{\begin{array}{ll}
\varphi^{j}\left[x_{i, 0}, x_{i, 1}, \ldots, x_{i, d}\right](x) & x \in I_{i} \\
0 & x \in \Omega \backslash I_{i}
\end{array}= \begin{cases}\prod_{0 \leq l \leq d, l \neq j} \frac{x-x_{i, l}}{x_{i, j}-x_{i, l}} & x \in I_{i} \\
0 & x \in \Omega \backslash I_{i} .\end{cases}\right.
$$

\subsubsection{Numerical scheme}

The function $f$ is projected at time $t^{n}$ on the basis $\left\{\varphi_{i, j}\right\}_{(i, j) \in\{0, \ldots, N-1\} \times\{0, \ldots, d\}}$ as

$$
f^{n}(x)=\sum_{i^{\prime}=0}^{N-1} \sum_{j^{\prime}=0}^{d} f_{i^{\prime}, j^{\prime}}^{n} \varphi_{i^{\prime}, j^{\prime}}(x)
$$

For a given initial function, the initialization is given by $f_{i, j}^{0}=f^{0}\left(x_{i, j}\right)$. Injecting the representation (4) into (3) leads to

$$
\begin{aligned}
\frac{\Delta x}{2} f_{i, j}^{n+1} \omega_{j} & =\sum_{i^{\prime}, j^{\prime}} f_{i^{\prime}, j^{\prime}}^{n} \int_{x_{i^{*}-1 / 2}+\alpha \Delta x}^{x_{i^{*}+1 / 2}} \varphi_{i^{\prime}, j^{\prime}}(x) \varphi_{i, j}(x+a \Delta t) d x \\
& +\sum_{i^{\prime}, j^{\prime}} f_{i^{\prime}, j^{\prime}}^{n} \int_{x_{i^{*}+1 / 2}}^{x_{i^{*}+1 / 2}+\alpha \Delta x} \varphi_{i^{\prime}, j^{\prime}}(x) \varphi_{i, j}(x+a \Delta t) d x
\end{aligned}
$$

where the index $i^{*}$ and the number $\alpha \in\left[0,1\left[\right.\right.$ are chosen such that $x_{i-1 / 2}-a \Delta t=x_{i^{*}-1 / 2}+\alpha \Delta x$. Their meaning is sketched in Figure 1. Then, using the fact that the basis functions $\varphi_{i, j}$ vanish outside the interval $I_{i}$, we get 


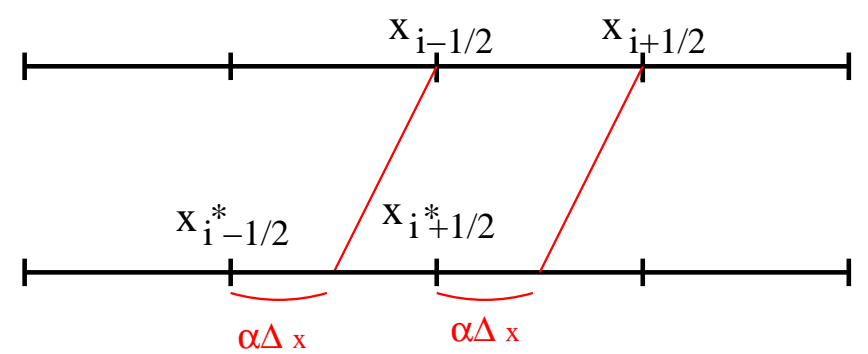

FIGURE 1. The meaning of the parameters $i^{*}$ and $\alpha$.

$$
\begin{aligned}
\frac{\Delta x}{2} f_{i, j}^{n+1} \omega_{j} & =\sum_{j^{\prime}} f_{i^{*}, j^{\prime}}^{n} \int_{x_{i^{*}-1 / 2}+\alpha \Delta x}^{x_{i^{*}+1 / 2}} \varphi_{i^{*}, j^{\prime}}(x) \varphi_{i, j}(x+a \Delta t) d x \\
& +\sum_{j^{\prime}} f_{i^{*}+1, j^{\prime}}^{n} \int_{x_{i^{*}+1 / 2}}^{x_{i^{*}+1 / 2}+\alpha \Delta x} \varphi_{i^{*}+1, j^{\prime}}(x) \varphi_{i, j}(x+a \Delta t) d x .
\end{aligned}
$$

Introducing the notation $\tilde{\varphi}^{j}(x)=\varphi^{j}\left[\tilde{\alpha}_{0}, \tilde{\alpha}_{1}, \ldots, \tilde{\alpha}_{d}\right](x)$ and the Gauß weigths $\omega_{j}$, the change of variables $x=$ $x_{i^{*}-1 / 2}+s \Delta x$ leads to

$$
f_{i, j}^{n+1}=\frac{2}{\omega_{j}} \sum_{j^{\prime}} f_{i^{*}, j^{\prime}}^{n} \int_{s=\alpha}^{1} \tilde{\varphi}^{j^{\prime}}(s) \tilde{\varphi}^{j}(s-\alpha) d s+\frac{2}{\omega_{j}} \sum_{j^{\prime}} f_{i^{*}+1, j^{\prime}}^{n} \int_{s=0}^{\alpha} \tilde{\varphi}^{j^{\prime}}(s) \tilde{\varphi}^{j}(s+1-\alpha) d s .
$$

Two integrals of $(2 d+1)$-order polynomials have to be evaluated. The changes of variables $s=\alpha+u(1-\alpha)$ in the first integral and $s=\alpha u$ in the second one enable to get

$$
\begin{aligned}
f_{i, j}^{n+1} & =\frac{2}{\omega_{j}} \sum_{j^{\prime}} f_{i^{*}, j^{\prime}}^{n}(1-\alpha) \int_{u=0}^{1} \tilde{\varphi}^{j^{\prime}}(\alpha+u(1-\alpha)) \tilde{\varphi}^{j}(u(1-\alpha)) d u \\
& +\frac{2}{\omega_{j}} \sum_{j^{\prime}} f_{i^{*}+1, j^{\prime}}^{n} \alpha \int_{u=0}^{1} \tilde{\varphi}^{j^{\prime}}(\alpha u) \tilde{\varphi}^{j}(\alpha(u-1)+1) d u,
\end{aligned}
$$

which is computed exactly by using the $d+1$ Gauß points and weigths

$$
\begin{aligned}
f_{i, j}^{n+1} & =\frac{1}{\omega_{j}} \sum_{j^{\prime}} f_{i^{*}, j^{\prime}}^{n}(1-\alpha) \sum_{r=0}^{d} \omega_{r} \tilde{\varphi}^{j^{\prime}}\left(\alpha+\tilde{\alpha}_{r}(1-\alpha)\right) \tilde{\varphi}^{j}\left(\tilde{\alpha}_{r}(1-\alpha)\right) \\
& +\frac{1}{\omega_{j}} \sum_{j^{\prime}} f_{i^{*}+1, j^{\prime}}^{n} \alpha \sum_{r=0}^{d} \omega_{r} \tilde{\varphi}^{j^{\prime}}\left(\alpha \tilde{\alpha}_{r}\right) \tilde{\varphi}^{j}\left(\alpha\left(\tilde{\alpha}_{r}-1\right)+1\right) .
\end{aligned}
$$

Remark: The factors 2 that appear are due to the fact that in our notations the weights $\omega_{r}$ are designed for the interval $[-1,1]$, and not for $[0,1]$, therefore we have to divide by its length which is 2 . As $L^{2}(\Omega)$-norm, $L^{1}(\Omega)$-norm and mass for the distribution function $f^{n}$ we shall use

$$
\left\|f^{n}\right\|_{L^{2}(\Omega)}^{2}=\frac{\Delta x}{2} \sum_{i, j} \omega_{j}\left(f_{i, j}^{n}\right)^{2}, \quad\left\|f^{n}\right\|_{L^{1}(\Omega)}=\frac{\Delta x}{2} \sum_{i, j} \omega_{j}\left|f_{i, j}^{n}\right|, \quad M\left(f^{n}\right)=\frac{\Delta x}{2} \sum_{i, j} \omega_{j} f_{i, j}^{n} .
$$

The scheme is by construction conservative: $\sum_{i, j} f_{i, j}^{n}=\sum_{i, j} f_{i, j}^{0}$. 


\subsection{Stability of the scheme}

In the context of linear advection, we study the amplification factor adapted to the case of the DG scheme, for periodic boundary conditions. We introduce as notation

$$
f_{k}=\left(f_{k, 0}, f_{k, 1}, \ldots, f_{k, d}\right) \in \mathbb{R}^{d+1}
$$

Using the notations introduced above, we define for $\left(j, j^{\prime}\right) \in\{0, \ldots, d\} \times\{0, \ldots, d\}$

$$
\begin{aligned}
\left(A_{0}\right)_{j, j^{\prime}} & =\frac{1}{\omega_{j}}(1-\alpha) \sum_{r=0}^{d} \omega_{r} \tilde{\varphi}^{j^{\prime}}\left(\alpha+\tilde{\alpha}_{r}(1-\alpha)\right) \tilde{\varphi}^{j}\left(\tilde{\alpha}_{r}(1-\alpha)\right) \\
\left(A_{1}\right)_{j, j^{\prime}} & =\frac{1}{\omega_{j}} \alpha \sum_{r=0}^{d} \omega_{r} \tilde{\varphi}^{j^{\prime}}\left(\alpha \tilde{\alpha}_{r}\right) \tilde{\varphi}^{j}\left(\alpha\left(\tilde{\alpha}_{r}-1\right)+1\right) \\
\left(A_{k}\right)_{j, j^{\prime}} & =0, \quad \forall k=2, \ldots N-1 .
\end{aligned}
$$

Without loss of generality, we shall suppose that the displacement is limited to one cell i.e. $0 \leq \alpha=-\frac{a \Delta t}{\Delta x}<1$.

It is obviously possible to use arbitrary time steps. In the numerical tests, we restrict the time step to the uniform mesh $\Delta x$ (and not to the Gauß points mesh). Since the present approach uses quite large $\Delta x$, this restriction is not important (compared to traditional schemes). Moreover, this makes the implementation easier and enables to use only neighboring data.

In this case the DG scheme can be written $f^{n+1}=A f^{n}$ where the matrix $A \in \mathcal{M}_{d+1}(\mathbb{R})$ is given by

$$
A=\left(\begin{array}{lllll}
A_{0} & A_{1} & 0 & \ldots & 0 \\
0 & A_{0} & A_{1} & \ddots & \vdots \\
\vdots & \ddots & \ddots & \ddots & 0 \\
0 & \ldots & 0 & A_{0} & A_{1} \\
A_{1} & 0 & \ldots & 0 & A_{0}
\end{array}\right) .
$$

We now introduce the Fourier transform adapted to vectorial coefficients of $\mathbb{R}^{d+1}$

$$
\begin{aligned}
\left(\mathbb{R}^{d+1}\right)^{N} & \longrightarrow\left(\mathbb{C}^{d+1}\right)^{N} \\
\left(f_{0}, f_{1}, \ldots, f_{N-1}\right) & \mapsto\left(\hat{f}_{0}, \hat{f}_{1}, \ldots, \hat{f}_{N-1}\right),
\end{aligned}
$$

with $\hat{f}_{k}=\sum_{j=0}^{N-1} e^{-2 i \pi k j / N} f_{j} \in \mathbb{C}^{d+1}$. Similarly, we define the Fourier transform $\hat{A}_{k}$ of the matrix $A: \hat{A}_{k}=$ $\sum_{j=0}^{N-1} e^{-2 i \pi k j / N} A_{j} \in \mathcal{M}_{d+1}(\mathbb{C})$. With these notations, we can then express the evolution of the Fourier modes of the solution

$$
\begin{aligned}
\hat{f}_{k}^{n+1} & =\sum_{\ell=0}^{N-1} f_{\ell}^{n+1} e^{-2 i \pi \ell k / N}=\sum_{\ell=0}^{N-1} \sum_{j=0}^{N-1} A_{\ell-j} f_{j}^{n} e^{-2 i \pi \ell k / N} \\
& =\sum_{j=0}^{N-1}\left(\sum_{\ell=0}^{N-1} A_{\ell-j} e^{-2 i \pi(\ell-j) k / N}\right) e^{-2 i \pi j k / N} f_{j}^{n}=\hat{A}_{k} \hat{f}_{k}^{n},
\end{aligned}
$$


where the indices are taken modulo $N$ since periodic conditions are considered. If we now diagonalize the matrix $\hat{A}_{k}$ by $\hat{A}_{k}=P_{k} \Delta_{k} P_{k}^{-1}$, then recursively $\hat{f}_{k}^{n}=P_{k}\left(\Delta_{k}\right)^{n} P_{k}^{-1} \hat{f}_{k}^{0}$. In our case, as $A_{\ell}=0$ for $\ell=2, \ldots, N-1$ we obtain $\hat{A}_{k}=A_{0}+A_{1} e^{-2 i \pi k / N}$. The amplification factor is then defined as $\rho(k / N)=\max _{0 \leq i \leq d}\left|\left(\Delta_{k}\right)_{i, i}\right|$. In order to have a bounded solution, the amplification factor should satisfy $\rho(\omega) \leq 1$ (or $1-\rho(\omega) \geq 0$ ), for $0 \leq \omega \leq 1$. We compare here this amplification factor with others schemes: centered Lagrange interpolation of degree 9 and 17 (LAG9,LAG17) (see [9,23]) and finite element interpolation of degree 1,2,3 and $4(Q 1, Q 2, Q 3$ and $Q 4)$. The amplification factor $(1-\rho(\omega))$ of these methods is plotted in Figure 2 for chosen values of $\omega=k / N$, as a function of the displacement $\alpha \in[0,1]$. Note that the finite element interpolation is unstable for a degree greater than 3 (see $Q 3$ and $Q 4$ in Figure 2). We also note that the DG schemes (in Figure 2, we have considered the schemes from degree 0 to 3: $D G 0, D G 1, D G 2, D G 3)$ remain stable and become less and less diffusive as the degree increases, as expected. We can also note that the scheme $D G 0$ coincides with the scheme $Q 1$. 

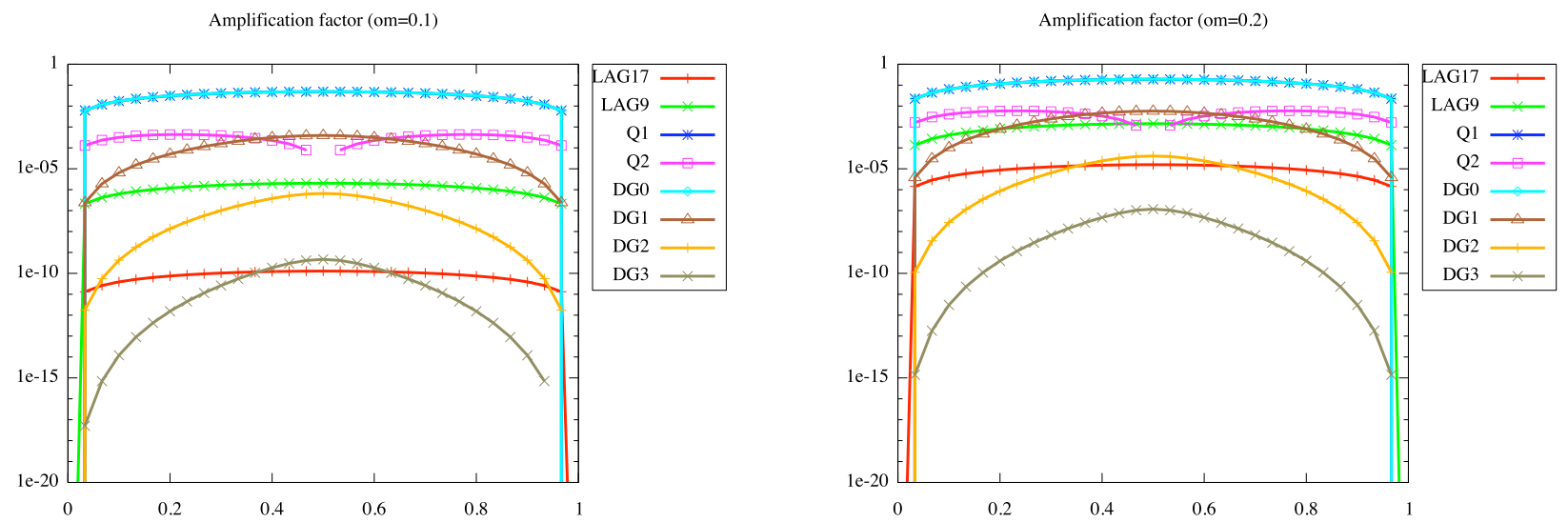

Amplification factor (om=0.3)
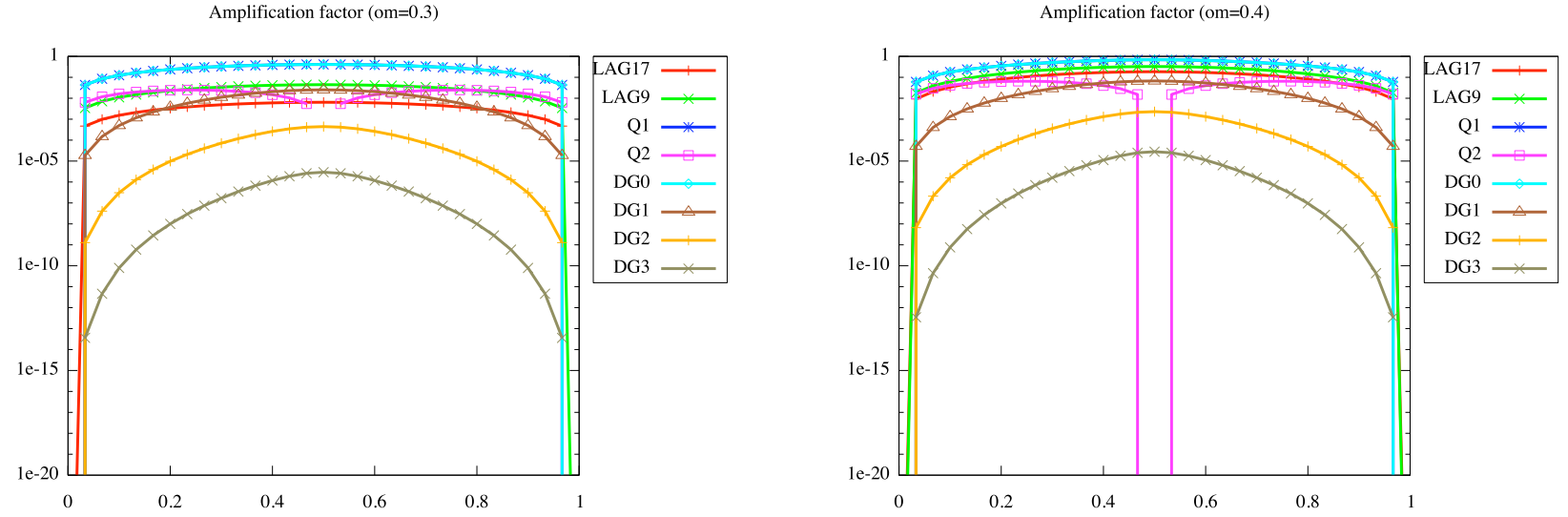

Amplification factor (om=0.5)

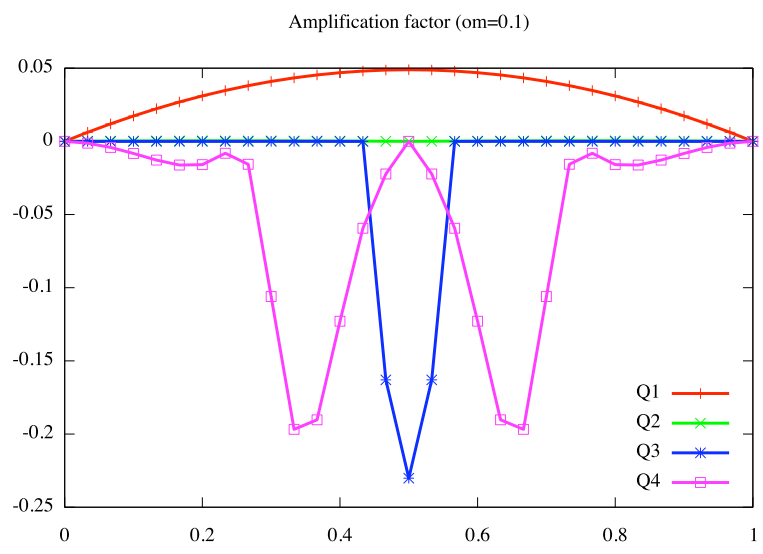

Figure 2. Amplification factor: $(1-\rho(\omega))$ is plotted for different values of $\omega(\omega=$ $0.1,0.2,0.3,0.4,0.5$ and again 0.1$)$ and different schemes, as a function of the normalized displacement $\alpha \in[0,1]$. 


\subsection{Extension to the Vlasov-Poisson problem}

The Vlasov-Poisson (1) problem involves a distribution function $f=f(t, x, v)$. Instead of solving a full $2 \mathrm{D}$ problem, we reduce to the solution of 1D problems of type (2) through the second-order Strang splitting scheme (see [6,23]). Obviously, higher order time splitting can be used (we refer to [14, 20, 24] for a discussion on this subject) but we restrict ourselves to second-order splitting to focus the attention on the influence of the phase-space discretization. Let us detail the global algorithm for the solving of the Vlasov-Poisson system.

The unknown quantities are then $f_{k, \ell, i, j}^{n}$ which are approximations of $f\left(t^{n}, x_{k, \ell}, v_{i, j}\right)$ where $x_{k, \ell}\left(\right.$ resp. $\left.v_{i, j}\right)$ corresponds to the $\ell$-th Gauß points in the cell $k$ (resp. $j$-th Gauß points in the cell $i$ ). We suppose periodic boundary conditions so that we only have to compute at each time $t^{n}$

$$
f_{k, \ell, i, j}^{n}, \text { for } k=0, \ldots, N_{x}-1, \ell=0, \ldots, d, \text { and } i=0, \ldots, N_{v}-1, j=0, \ldots, d,
$$

on each Gauß point of each cell. By denoting the transport operator $\mathcal{T}_{\alpha}^{x}$ in the $x$-direction (or $\mathcal{T}_{\alpha}^{v}$ in the $v$-direction) described in the previous section, the time-splitting algorithm then reads (see [6])

Step 0. Initialization: $f_{k, \ell, i, j}=f^{0}\left(x_{k, \ell}, v_{i, j}\right), k=0, \ldots, N_{x}-1, \ell=0, \ldots, d, i=0, \ldots, N_{v}-1, j=0, \ldots, d$.

Step 1. Half time step shift along the $x$-axis:

For each $i=0, \ldots, N_{v}-1, j=0, \ldots, d,\left(f_{k, \ell, i, j}\right)_{k=0, \ell=0}^{N_{x}-1, d} \rightarrow \mathcal{T}_{\alpha}^{x}\left(\left(f_{k, \ell}\right)_{k=0, \ell=0}^{N_{x}-1, d}\right)$ with $\alpha=-v_{i, j} \Delta t / 2$.

Step 2. Computation of the charge density $\rho_{k, \ell}$ and of the electric field by integrating

the Poisson equation $\partial_{x} E=\rho-1$ by using the method proposed in Appendix A.

Step 3. Shift along the $v$-axis:

For each $k=0, \ldots, N_{x}-1, \ell=0, \ldots, d,\left(f_{k, \ell, i, j}\right)_{i=0, j=0}^{N_{v}-1, d} \rightarrow \mathcal{T}_{\alpha}^{v}\left(\left(f_{k, \ell, i, j}\right)_{i=0, j=0}^{N_{v}-1, d}\right)$ with $\alpha=-E_{k, \ell} \Delta t$.

Step 4.a Half time step shift along the $x$-axis:

For each $i=0, \ldots, N_{v}-1, j=0, \ldots, d,\left(f_{k, \ell, i, j}\right)_{k=0, \ell=0}^{N_{x}-1, d} \rightarrow \mathcal{T}_{\alpha}^{x}\left(\left(f_{k, \ell, i, j}\right)_{k=0, \ell=0}^{N_{x}-1, d}\right)$ with $\alpha=-v_{i, j} \Delta t / 2$.

Step 4.b We have $f_{k, \ell, i, j}^{n}=f_{k, \ell, i, j}$, for $k=0, \ldots, N_{x}-1, \ell=0, \ldots, d, i=0, \ldots, N_{v}-1, j=0, \ldots, d$.

Step 4.c Half time step shift along the $x$-axis:

For each $i=0, \ldots, N_{v}-1, j=0, \ldots, d,\left(f_{k, \ell, i, j}\right)_{k=0, \ell=0}^{N_{x}-1, d} \rightarrow \mathcal{T}_{\alpha}^{x}\left(\left(f_{k, \ell, i, j}\right)_{k=0, \ell=0}^{N_{x}-1, d}\right)$ with $\alpha=-v_{i, j} \Delta t / 2$.

Step 5. $n \rightarrow n+1$ and loop to Step 2 .

Note that if we make no diagnostic of the distribution function, we can simplify Step 4.a-c into

Step 4. Shift along the $x$-axis:

For each $i=0, \ldots, N_{v}-1, j=0, \ldots, d,\left(f_{k, \ell, i, j}\right)_{k=0, \ell=0}^{N_{x}-1, d} \rightarrow \mathcal{T}_{\alpha}^{x}\left(\left(f_{k, \ell, i, j}\right)_{k=0, \ell=0}^{N_{x}-1, d}\right)$ with $\alpha=-v_{i, j} \Delta t$.

\section{NUMERICAL EXPERIMENTS}

This section is devoted to the numerical experiments of the new method. First, tests are performed on the linear advection, on which order in space can be verified. Then, the extension to the Vlasov-Poisson case is tackled. Some comparisons with the semi-Lagrangian method with cubic splines interpolation are also presented.

\subsection{D linear advection}

To validate the implementation, the linear advection with periodic boundary conditions is studied

$$
\partial_{t} f+\partial_{x} f=0, \quad t \geq 0, \quad x \in[0,1] .
$$

For a given initial condition $f_{0}(x), x \in[0,1]$, the numerical solution is compared to the analytical one $f(t, x)=$ $f_{0}(x-t), \forall t \geq 0$.

Two different initial profiles (a regular and a discontinuous one) are considered to verify the order-in-space of the method. As diagnostics, the $L^{p}$-norms $(p=1,2, \infty)$ of the difference between the numerical and the analytical solutions are plotted in Figure 3 and Figure 4 (the errors are plotted as a function of the number of points in log-log scale). We observe that for a smooth initial profile, the order of the method is greater than 
$(d+1)$; it is not true for the discontinuous case since the method is of order 1 for every degree of the basis of the DG space. The numerically computed slopes are given in the associated figures.
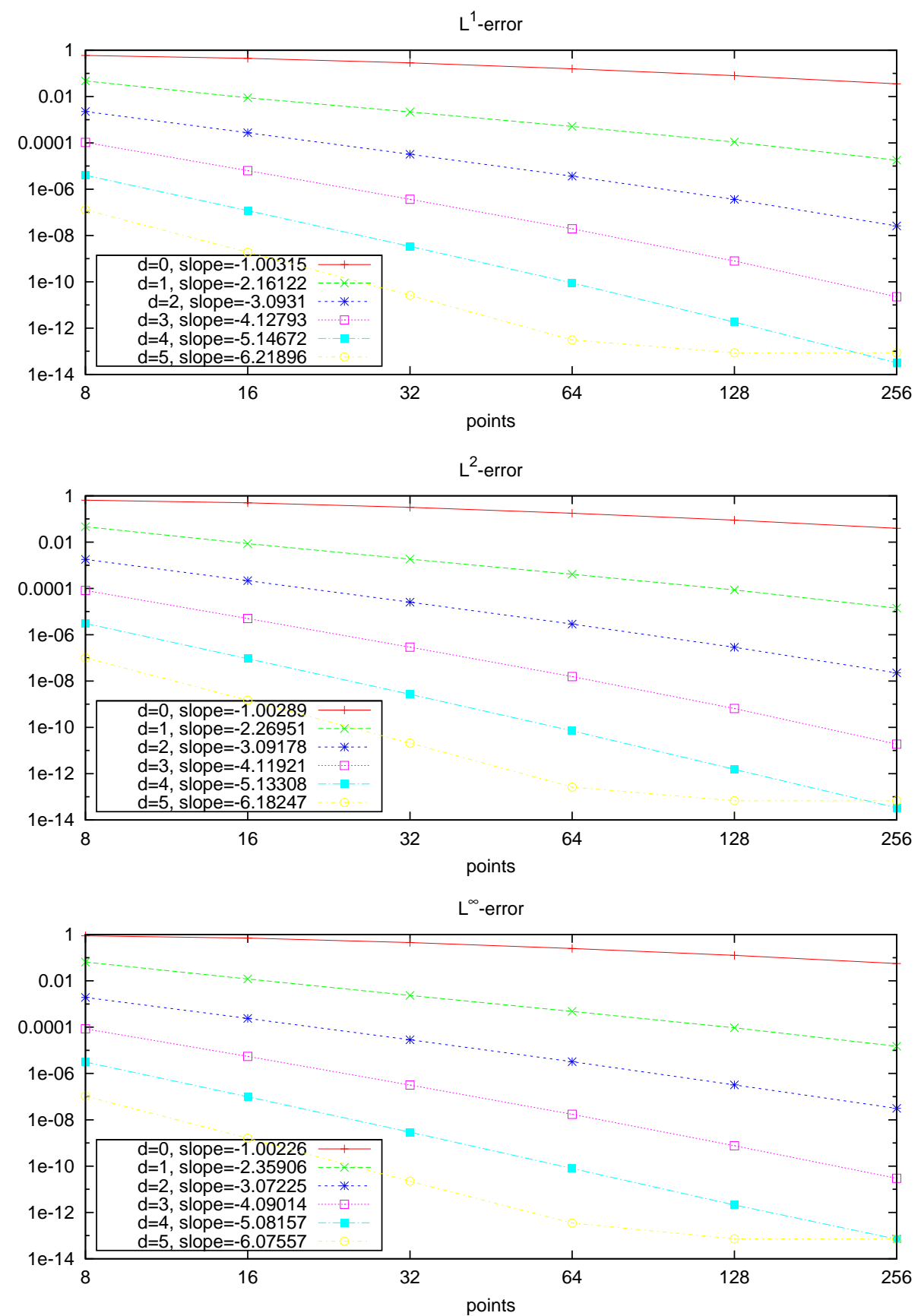

Figure 3. 1D linear advection. The $L^{1}$, the $L^{2}$ and the $L^{\infty}$ errors as a function of the number of points, committed with respect to the exact solution, for the initial function $f^{0}(x)=$ $\sin (2 \pi x-\pi)$, in the interval $[0,1]$ with periodic boundary conditions. 

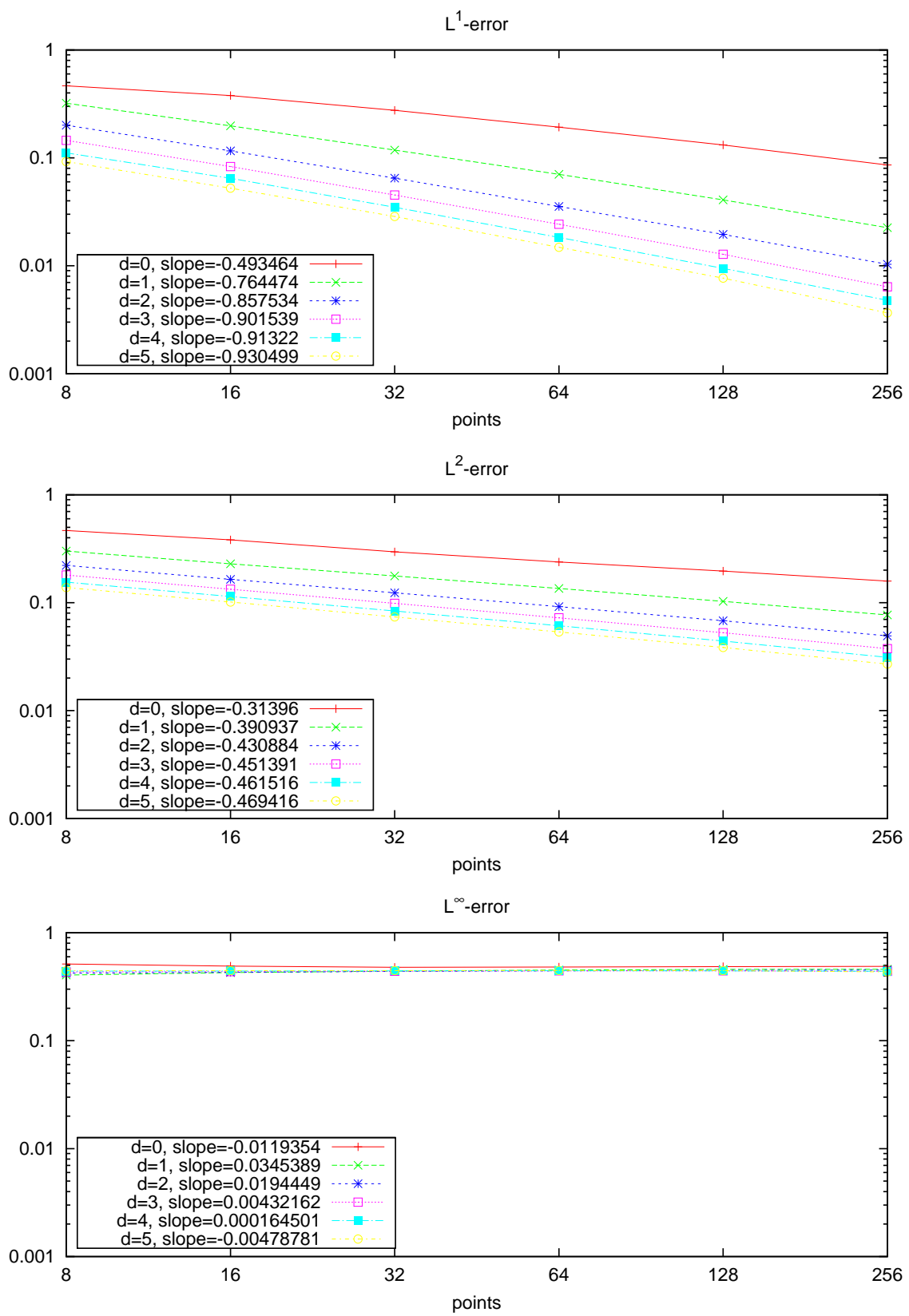

Figure 4. 1D linear advection. The $L^{1}$, the $L^{2}$ and the $L^{\infty}$ errors as a function of the number of points, committed with respect to the exact solution, for the initial function $f^{0}(x)=$ $\chi_{\left[0, \frac{1}{2}\right]}(x)$, in the interval $[0,1]$ with periodic boundary conditions.

\subsection{One-dimensional Landau damping}

We are now interested in the numerical solution of the initial value Vlasov-Poisson problem

$$
\left\{\begin{array}{l}
\partial_{t} f+v \partial_{x} f+E \partial_{v} f=0, \quad \partial_{x} E=\int_{\mathbb{R}} f d v-1, \\
f_{0}(x, v)=\frac{e^{-v^{2} / 2}}{\sqrt{2 \pi}}(1+\alpha \cos (k x)) .
\end{array}\right.
$$


For the linear regime $(\alpha=0.001)$, we consider the numerical phase-space interval $(x, v) \in[0,2 \pi / k] \times[-6,6]$. For the nonlinear regime $(\alpha=0.5)$, we choose $(x, v) \in[0,2 \pi / k] \times[-9,9]$.

The algorithm is based on a Strang splitting procedure so that the $x$ and $v$-advections are nothing else but the linear advections validated just above. Between two successive advections, the electric field has to be computed on the degrees of freedom so that a standard spectral solver cannot be easily used. Details about the computation of the electrostatic field are done in Appendix A.

Linear regime. The DG method is running with $N_{x}=N_{v}=30$ points and 5 Gauß points per cell enable to reconstruct a 4 th order polynomial. In the linear regime, the $L^{2}$-norm of the electric field is known to decay exponentially in time, the rate of which can be computed a priori (see [8,21]). In Table 1 the numerical decay rate together with the period of the oscillations are presented, for different values of the initial mode $k$. We observe that they are in a very good agreement with the linear theory.

Nonlinear regime. For the nonlinear case ( $\alpha=0.5$ in the initial condition), the linear theory cannot be applied so that the validation is performed through the conserved quantities of the model or by comparing DG with methods available in the literature.

In Figure 5, the evolution of the distribution function is plotted together with its space averaging

$\int_{0}^{2 \pi / k} f(t, x, v) d x$. We can observe that the method is able to capture the typical filamentation in the phase-space and nevertheless to remain stable. After large time, the velocity distribution, which presents strong oscillations during the evolution, is finally smoothed, as well as the distribution function itself which presents trapped particles on its tail.

In Figure 6 the DG method is compared to the backward semi-Lagrangian method with cubic spline reconstruction (BSL). To do this, the numerical parameters are chosen as follows: for BSL $N_{x}=N_{v}=150$ and we make vary the degree for the DG method such that the product $(d+1) \times N_{x}=(d+1) \times N_{v}$ is nearly constant equal to 150 , with $d=2,3,4,5\left(d=2, N_{x}=50 ; d=3, N_{x}=38 ; d=4, N_{x}=30 ; d=5, N_{x}=25\right)$. As diagnostics, we consider the electric energy (in log scale) and the time history of conserved quantities $\left(L^{1}\right.$ and $L^{2}$-norms).

First, in Figure 6, we can observe the good behavior of the present method compared to BSL. As the degree increases, we observe that the $L^{2}$-norm is becoming closer and closer to that of BSL which is well known to be very little diffusive. The $L^{2}$-norm decreases with time since, as observed before, the small structures are diffused when they become lower to the size of the mesh. These observation are compatible with results of the literature (see $[5,7,11])$. The $L^{1}$-norm is not preserved since, as we deal with high-order methods, some negative values are created; however, this is of lower importance compared to the BSL method.

Finally, in Figure 7 we show how the increase in the order-in-space $d\left(N_{x}\right.$ and $N_{v}$ are fixed to 30) of the method improves the resolution of the filamentation. This relatively easy increase of the order-in-space is one of the advantages of the approach. Obviously, the results are improved by increasing $d$. 


\begin{tabular}{||l||l|l||}
\hline$k$ & $\alpha=0.001$ (linear) & $\alpha=0.5$ (nonlinear) \\
\hline \hline 0.2 & $\begin{array}{l} \pm 1.07154+6.81267 \times 10^{-5} i \\
\left( \pm 1.0640-5.51 \times 10^{-5} i\right)\end{array}$ & $\pm 1.09402-0.00107607 i$ \\
\hline 0.3 & $\begin{array}{l} \pm 1.16209-0.0124224 i \\
( \pm 1.1598-0.0126 i)\end{array}$ & $\pm 1.30507-0.128511 i$ \\
\hline 0.4 & $\begin{array}{l} \pm 1.28645-0.0659432 i \\
( \pm 1.2850-0.0661 i)\end{array}$ & $\pm 1.3581-0.205133 i$ \\
\hline 0.5 & $\begin{array}{l} \pm 1.41696-0.152849 i \\
( \pm 1.4156-0.1533 i)\end{array}$ & $\pm 1.47343-0.279512 i$ \\
\hline
\end{tabular}

TABLE 1. 1D linear Landau damping. The decay rate and period of the oscillations of the electric field in the linear Landau problem, and its comparisons with the results in [21] (in the parentheses). Here, $d=4, N_{x} \times N_{v}=30 \times 30$. The time step is chosen by the method itself in order to limit the displacements to one cell. 

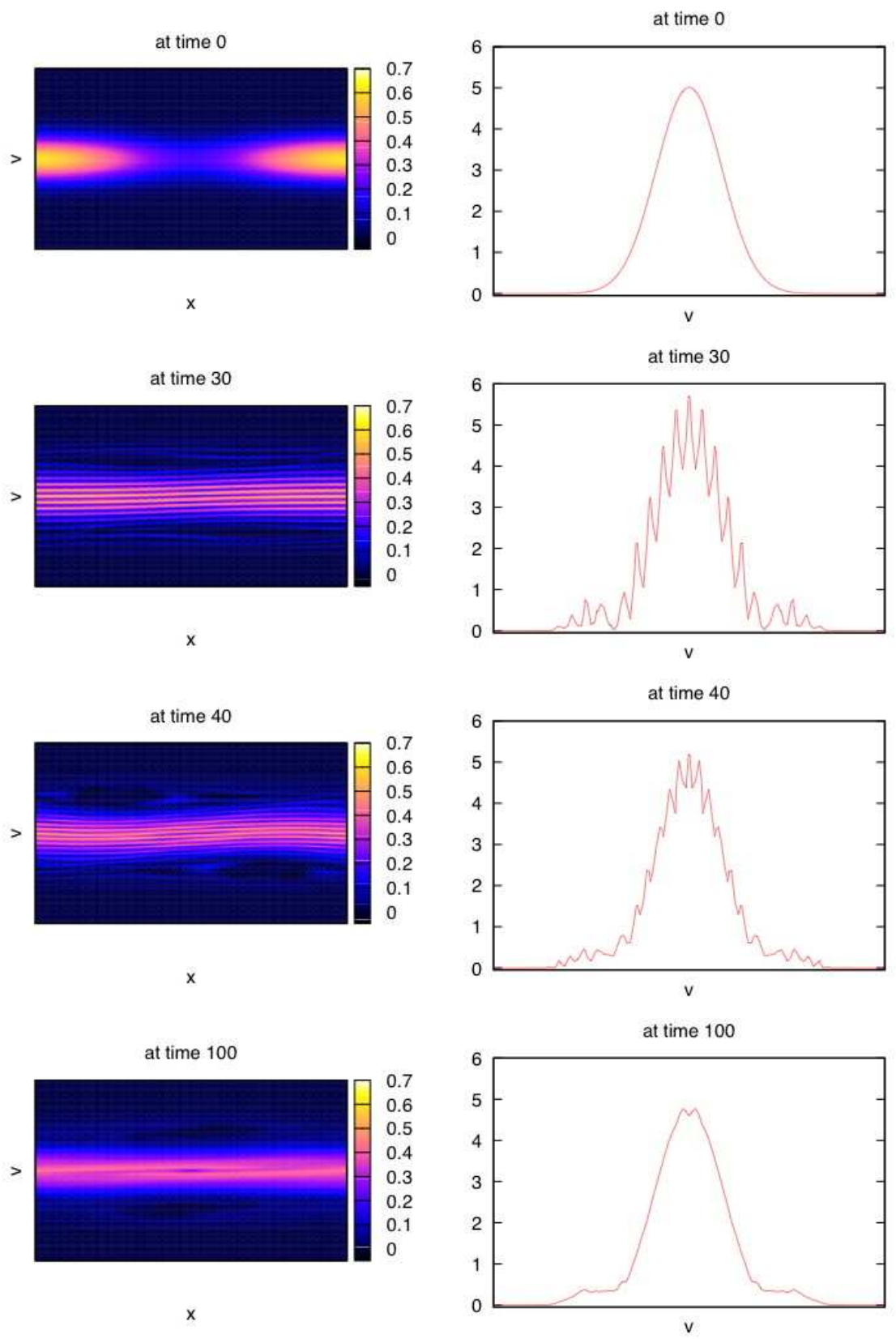

Figure 5. Strong Landau damping. The evolution of the distribution function and the velocity distribution for the nonlinear Landau damping. Here $d=4, N_{x} \times N_{v}=30 \times 30$, the time step $\Delta t$ is chosen by the method itself in order to limit the displacement to one cell. 

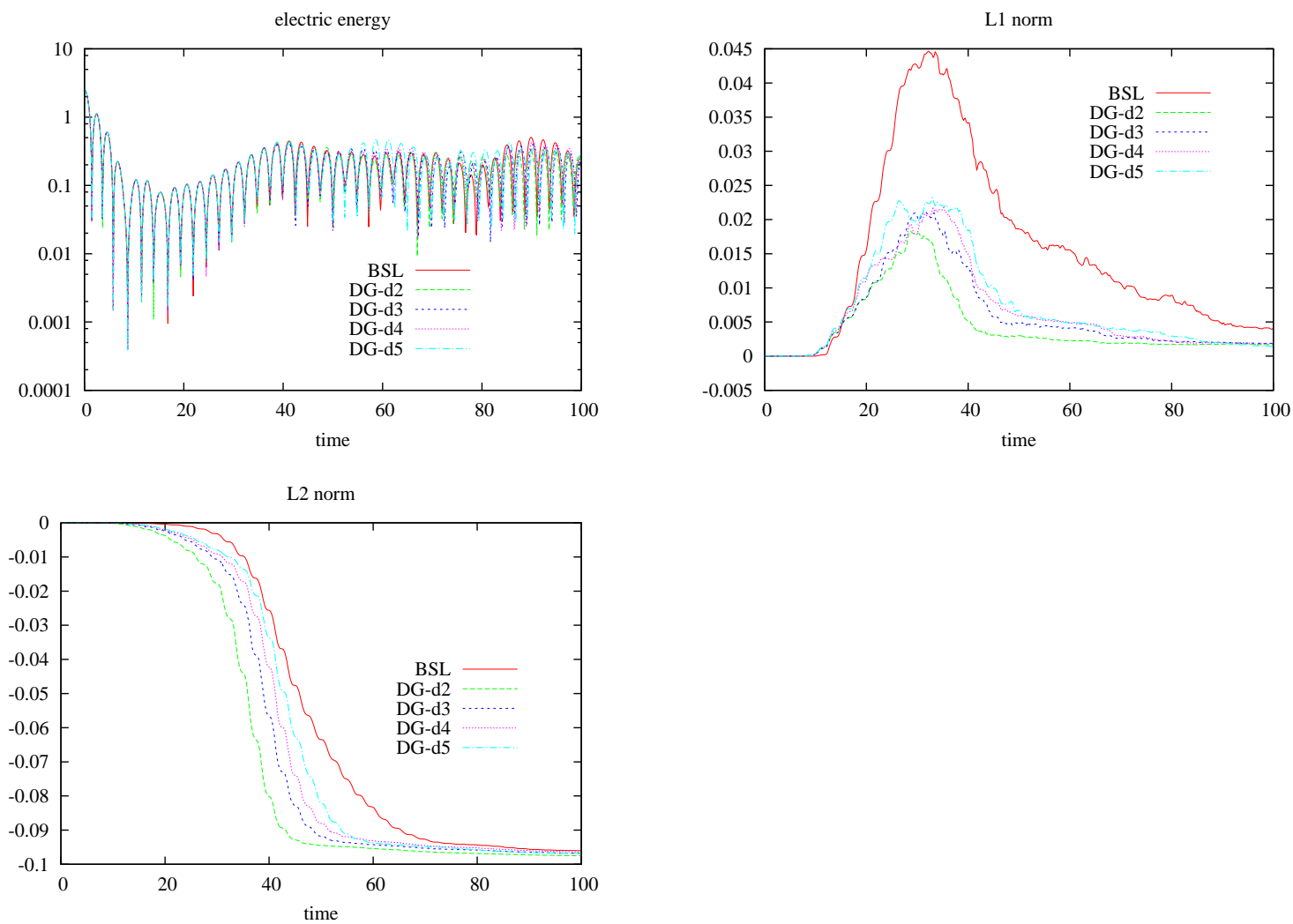

FiguRE 6. Strong Landau damping. The time evolution of the scalar magnitudes for the strong Landau damping test with a cubic splines reconstruction and DG method. Here $N_{x}=N_{v}=150$ for BSL and $d, N_{x}=N_{v}$ such that $(d+1) \times N_{x}=(d+1) \times N_{v}$ is nearly fixed to 150 for DG $(d=2,3,4,5)$. The time step is $\Delta t=0.1$ for both methods. Left: electric energy (log scale); middle: $L^{1}$-norm; right: $L^{2}$-norm. 
$d=1$, time $=25$

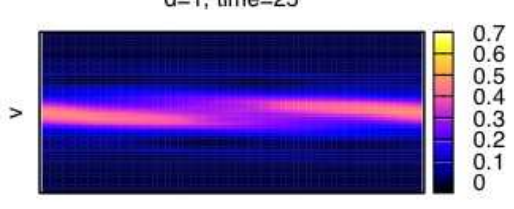

$x$

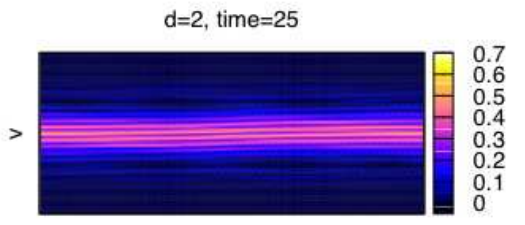

$\mathrm{x}$

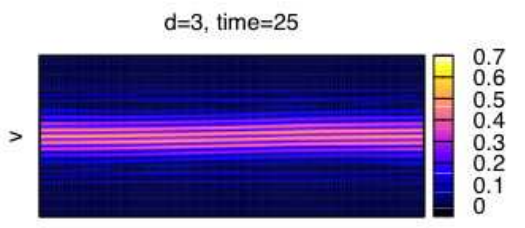

$\mathrm{x}$

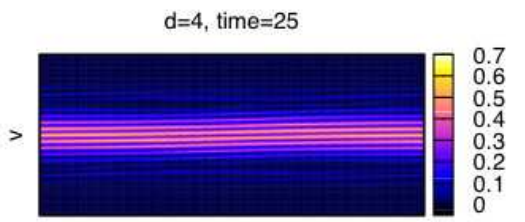

$\mathrm{x}$

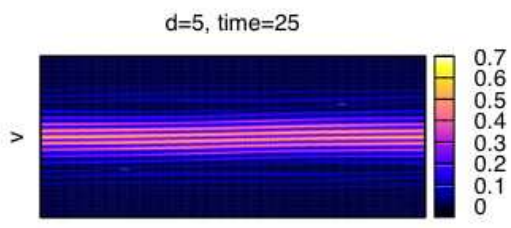

$x$ $\mathrm{d}=1$, time $=50$

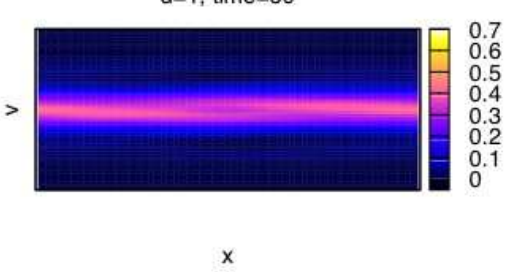

$d=2$, time $=50$

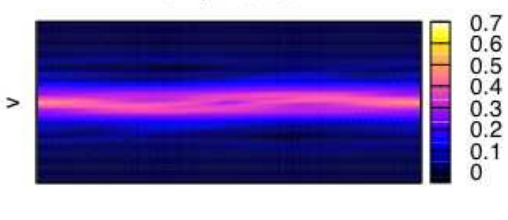

$\mathrm{x}$

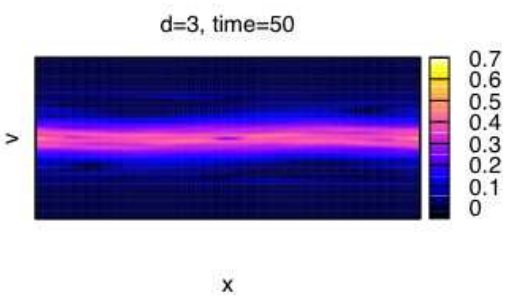

$d=4$, time $=50$

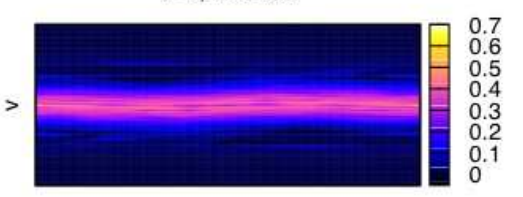

$\mathrm{x}$

$d=5$, time $=50$

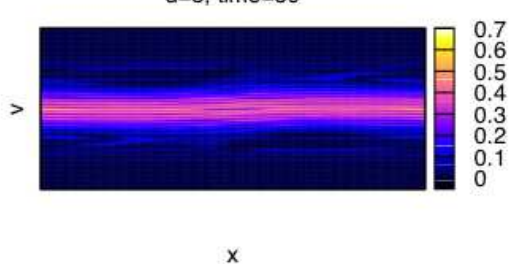

Figure 7. Strong Landau damping. DG method: study of the resolution of the filamentation of the phase-space for different values of $d$. Here $N_{x}=N_{v}=30$. 


\subsection{The Bump-On-Tail problem}

The Vlasov-Poisson equation is also solved, but the initial condition is chosen as follows (see [18,22]):

$$
f_{0}(x, v)=\frac{9}{10 \sqrt{2 \pi}} e^{-v^{2} / 2}+\frac{2}{10 \sqrt{2 \pi}} e^{-2|v-4.5|^{2}}(1+0.03 \cos (0.3 x))
$$

in the interval $(x, v) \in[0,20 \pi] \times[-9,9]$. For this system, an instability is expected so that three vortices are created which travel periodically in the phase-space. This problem is a challenging benchmark because it requires the solver to be accurate to describe the filamentation and to keep the three vortices separated. In Figure 8, we sketch the evolution of the distribution function in the phase-space as well as the evolution of the electric energy. For this latter quantity, the maximum is reached at $t \approx 20$ (after the linear part), and a periodic behavior can be observed, on which fast oscillations are superimposed. This is in very good agreement with the results in the literature $[7,18,22]$.

Results obtained by a cubic splines interpolation (BSL) are also presented for comparison (see Figure 9), with $N_{x}=N_{v}=150$ and $\Delta t=0.1$. The methods are very close. Keeping fixed the quantity $(d+1) \times N_{x}=(d+1) \times N_{v}$ in the DG methods, we can compare with the BSL method. We can observe that $d=3$ leads to the merging of vortices (breaking of the oscillating behavior of the electric energy at $t \approx 320$ ). When the degree increases however, this breaking disappears and the behavior is comparable to that of BSL. Note that the breaking which occurs for $d=5, N_{x}=N_{v}=25$ is explained by the low number of cells; by increasing it to $N_{x}=N_{v}=30$, the breaking can be pushed back (see Figure 8). The $L^{2}$-norm is well conserved (in a better way compared to BSL for this test case); as previously, we observe that the positivity is not ensured since the $L^{1}$-norm is not preserved (it is less preserved compared to BSL). However, the total energy is quite well preserved compared to BSL, even with $d=2$. Obviously, slope limiters could be added to ensure positivity, but the conservation of the other invariants (total energy and $L^{2}$-norm) may then deteriorate (see [7]).

\section{Conclusions}

We have shown that DG schemes are a valid tool to solve kinetic problems, and set the basis for their use in more complicated problems. The order of the method has been carefully validated against analytical solution but also against BSL on classical test cases of plasma physics through the Vlasov-Poisson model.

Natural extensions of this approach consist in the non constant advection case which arises in gyrokinetic models for example. Furthermore, slope limiters to ensure positivity or to limit the spurious oscillations can be introduced as in $[26]$.

Obviously, using a directional splitting, higher dimensional models can be envisaged (as in [13]). The extension of higher dimensional Poisson equation is indeed not so trivial (obviously, the present strategy cannot be used). Several strategies can be envisaged. A first one consists in designing an elliptic solver, whose solution is the electric potential at the Gauß points (as in $[1,2,20]$ ). Another strategy consists in a change of basis functions: a projection operator from the DG basis to a spectral one enables to compute the electric field $E$ using a spectral solver; then $E$ is evaluated on the Gauß points (using its representation in the Fourier space) for the following velocity advection. Fast algorithms and pre-computations can be performed so that these steps are not so expensive. This kind of algorithm is used in a work in preparation of the present authors.

\section{A. Solving the Poisson equation}

We propose a simple method to solve the Poisson equation $\partial_{x} E=\rho$ with the constraint $\int_{0}^{L} E(t, x) d x=0$, to get the electric field at the Gauß points. The neutrality constraint $\int_{0}^{L} \rho(x) d x=0$ corresponds to periodicity conditions on the electric field $E(L)=E(0)$. 
at time 0

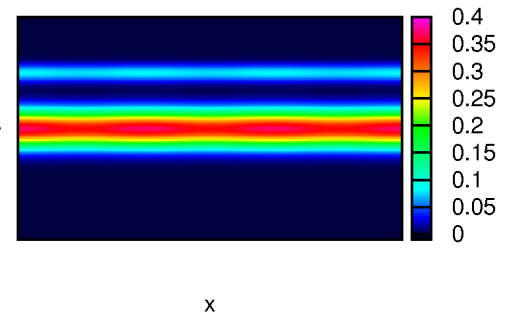

at time 20

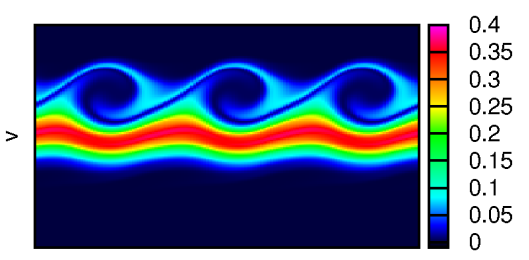

$\mathrm{x}$

at time 40

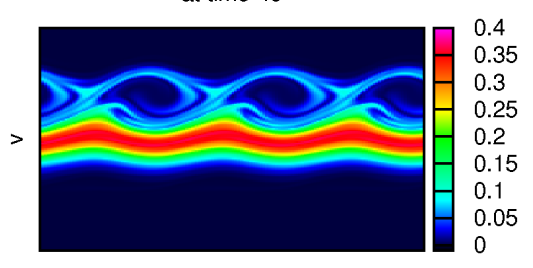

$x$

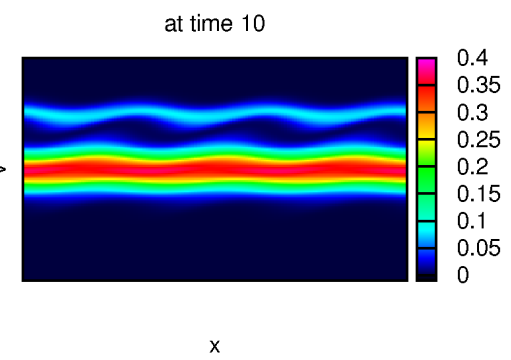

at time 30

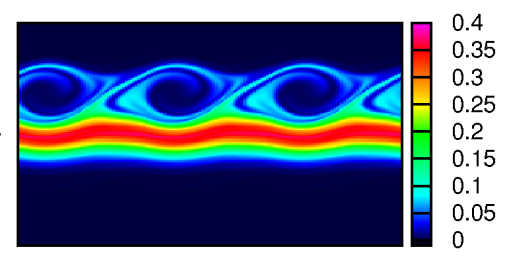

$\mathrm{x}$

at time 400

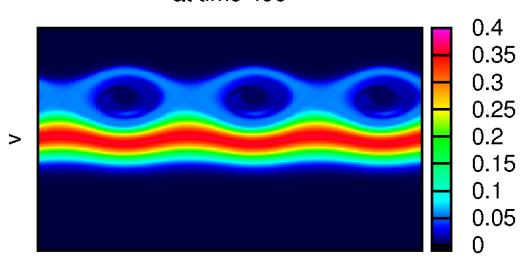

$x$

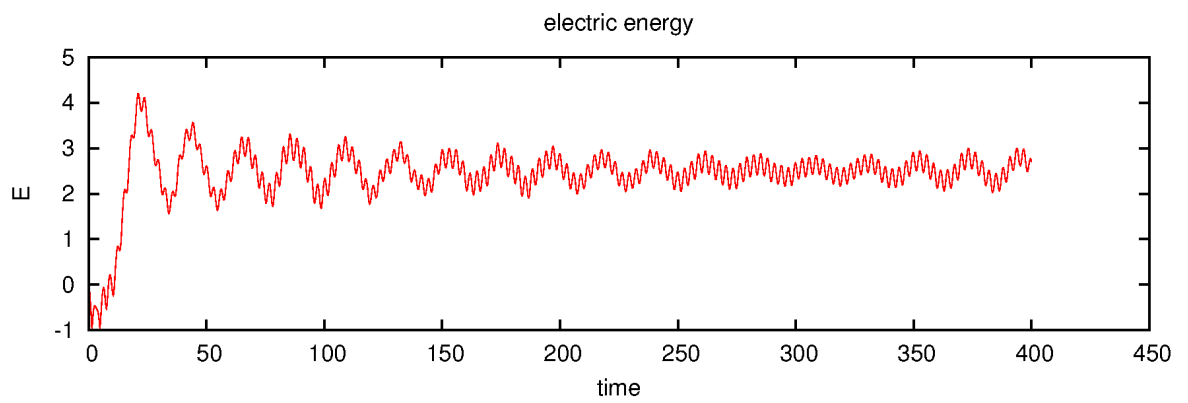

Figure 8. Bump-On-Tail. The evolution of the distribution functions for the Bump-OnTail, and of the electric energy. Here $d=5, N_{x} \times N_{v}=30 \times 30$, the time step $\Delta t$ is chosen by the method itself in order to limit the displacement to one cell.

The solution to the $1 D$ Poisson equation is given by

$$
E(x)=\int_{0}^{L} K(x, y)[\rho(y)-1] d y=\frac{1}{L} \int_{0}^{L} y \rho(y) d y-\int_{x}^{L} \rho(y) d y
$$



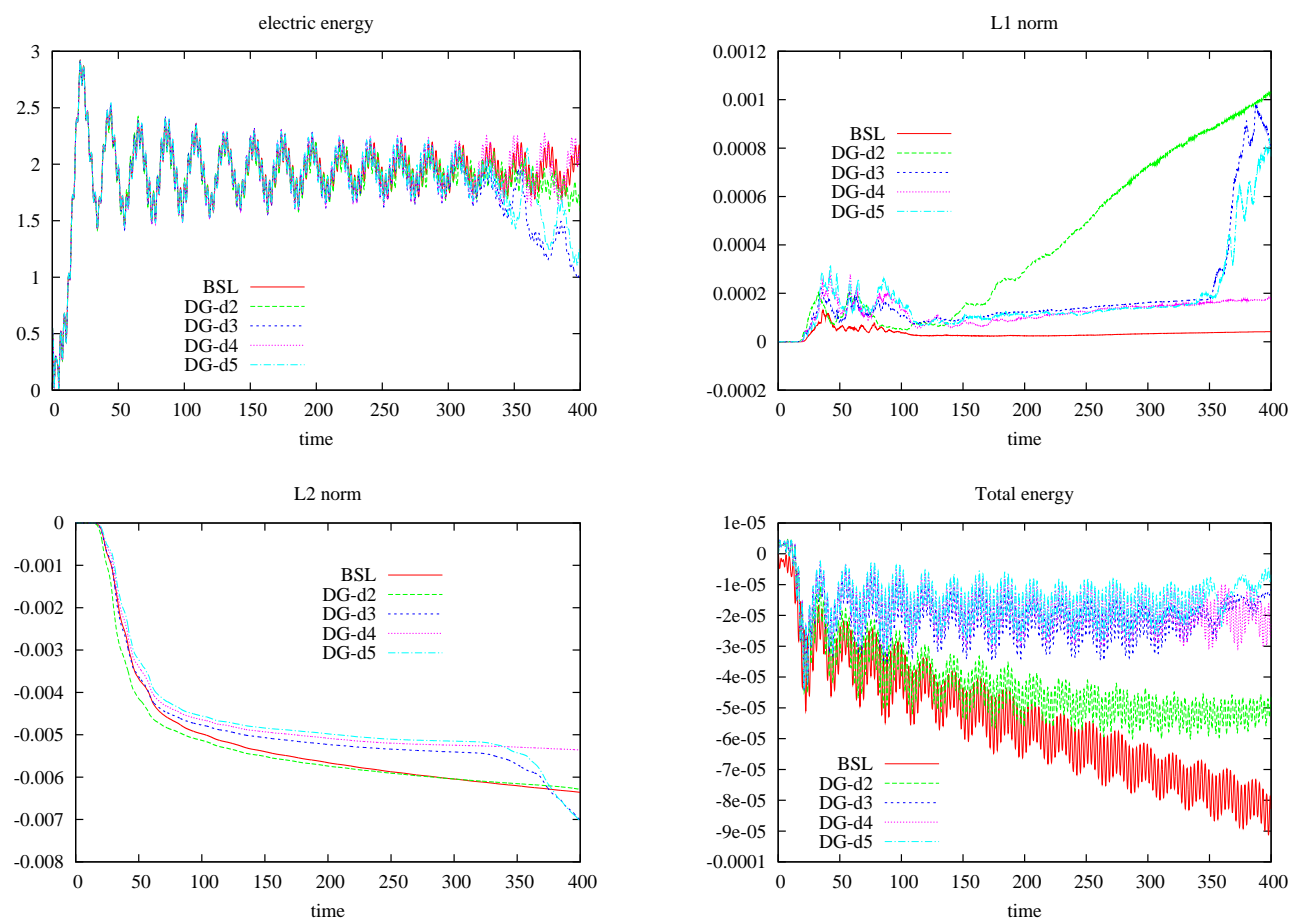

Figure 9. Bump-On-Tail. The time evolution of the scalar magnitudes for the Bump-OnTail with a BSL and DG method. Here $N_{x}=N_{v}=150$ for BSL and $(d+1) \times N_{x}=(d+1) \times N_{v}$ is kept nearly constant equal to 150: $d=2, N_{x}=N_{v}=50 ; d=3, N_{x}=N_{v}=38 ; d=4, N_{x}=$ $N_{v}=30 ; d=5, N_{x}=N_{v}=25$. The time step is $\Delta t=0.1$ for both methods. Top left: electric energy; top right: $L^{1}$-norm; bottom left: $L^{2}$-norm; bottom right: total energy.

where $K$ is the Green kernel

$$
K(x, y)= \begin{cases}\frac{y}{L} & y \in(0, x), \\ \frac{y}{L}-1 & y \in(x, L) .\end{cases}
$$

Discretization. - The density $\rho$ is represented on the DG basis by $\rho(x)=\sum_{i, j} \rho_{i, j} \varphi_{i, j}(x)$. Then,

$$
\begin{aligned}
E_{i, j} & =\frac{1}{L} \frac{\Delta x}{2} \sum_{i^{\prime}, j^{\prime}} x_{i^{\prime}, j^{\prime}} \omega_{j^{\prime}} \rho_{i^{\prime}, j^{\prime}}-\int_{x_{i, j}}^{x_{i+1 / 2}} \rho(y) d y-\int_{x_{i+1 / 2}}^{L} \rho(y) d y \\
& =\frac{1}{L} \frac{\Delta x}{2} \sum_{i^{\prime}, j^{\prime}} x_{i^{\prime}, j^{\prime}} \omega_{j^{\prime}} \rho_{i^{\prime}, j^{\prime}}-\int_{x_{i, j}}^{x_{i+1 / 2}} \rho(y) d y-\frac{\Delta x}{2} \sum_{i^{\prime}=i+1}^{N-1} \sum_{j^{\prime}=0}^{d} \omega_{j^{\prime}} \rho_{i^{\prime}, j^{\prime}} .
\end{aligned}
$$


We still have to treat the term $\int_{x_{i, j}}^{x_{i+1 / 2}} \rho(y) d y$ :

$$
\begin{aligned}
\int_{x_{i, j}}^{x_{i+1 / 2}} \rho(y) d y & =\int_{x_{i, j}}^{x_{i+1 / 2}} \sum_{i^{\prime}, j^{\prime}} \rho_{i^{\prime}, j^{\prime}} \varphi_{i^{\prime}, j^{\prime}}(y) d y=\sum_{i^{\prime}, j^{\prime}} \rho_{i^{\prime}, j^{\prime}} \int_{x_{i, j}}^{x_{i+1 / 2}} \varphi_{i^{\prime}, j^{\prime}}(y) d y \\
& =\sum_{j^{\prime}} \rho_{i, j^{\prime}} \int_{x_{i, j}}^{x_{i+1 / 2}} \varphi_{i, j^{\prime}}(y) d y=\sum_{j^{\prime}} \rho_{i, j^{\prime}} \Delta x \int_{s=\tilde{\alpha}_{j}}^{1} \tilde{\varphi}^{j^{\prime}}(s) d s \quad\left(\text { with } y=x_{i-1 / 2}+s \Delta x\right) \\
& =\sum_{j^{\prime}} \rho_{i, j^{\prime}} \Delta x\left(1-\tilde{\alpha}_{j}\right) \int_{u=0}^{1} \tilde{\varphi}^{j^{\prime}}\left(\tilde{\alpha}_{j}+u\left(1-\tilde{\alpha}_{j}\right)\right) d u \quad\left(\text { with } s=\tilde{\alpha}_{j}+u\left(1-\tilde{\alpha}_{j}\right)\right) \\
& =\frac{\Delta x}{2}\left(1-\tilde{\alpha}_{j}\right) \sum_{j^{\prime}} \rho_{i, j^{\prime}} \sum_{r=0}^{d} \omega_{r} \tilde{\varphi}^{j^{\prime}}\left(\tilde{\alpha}_{j}+\tilde{\alpha}_{r}\left(1-\tilde{\alpha}_{j}\right)\right)
\end{aligned}
$$

We finally have obtained the following expression for the electric field at the Gauß points

$$
E_{i, j} \approx \frac{\Delta x}{2 L} \sum_{i^{\prime}, j^{\prime}} x_{i^{\prime}, j^{\prime}} \omega_{j^{\prime}} \rho_{i^{\prime}, j^{\prime}}-\frac{\Delta x}{2}\left(1-\tilde{\alpha}_{j}\right) \sum_{j^{\prime}} \rho_{i, j^{\prime}} \sum_{r=0}^{d} \omega_{r} \tilde{\varphi}^{j^{\prime}}\left(\tilde{\alpha}_{j}+\tilde{\alpha}_{r}\left(1-\tilde{\alpha}_{j}\right)\right)-\frac{\Delta x}{2} \sum_{i^{\prime}=i+1}^{N-1} \sum_{j^{\prime}=0}^{d} \omega_{j^{\prime}} \rho_{i^{\prime}, j^{\prime}} .
$$

\section{REFERENCES}

[1] B. Ayuso de Dios, J. A. Carrillo, C.-W. Shu, Discontinuous Galerkin methods for the multi-dimensional Vlasov-Poisson problem, Preprint UAB.

[2] B. Ayuso, J. A. Carrillo, C.-W. Shu, Discontinuous Galerkin Methods for the one-dimensional Vlasov-Poisson System, Preprint UAB.

[3] R. Bermejo, Analysis of an algorithm for the Galerkin-characteristic method, Numer. Math. 60, pp. 163-194, (1991).

[4] C.K. Birdsall, A.B. Langdon, Plasma Physics via Computer Simulation, Inst. of Phys. Publishing, Bristol/Philadelphia, 1991.

[5] J.A. Carrillo, F. Vecil, Non oscillatory interpolation methods applied to Vlasov-based models, SIAM Journal of Scientific Computing 29, 1179-1206, 2007.

[6] C.Z. Cheng And G. Knorr, The integration of the Vlasov equation in configuration space, J. Comput. Phys. 22, pp. 330-351, (1976).

[7] N. Crouseilles, M. Mehrenberger, E. SonnendrüCker, Conservative semi-Lagrangian schemes for the Vlasov equation, J. Comput. Phys. 229, (2010), pp 1927-1953.

[8] N. Crouseilles, T. Respaud, E. Sonnendrücker, A forward semi-Lagrangian method for the numerical solution of the Vlasov equation, Comput. Phys. Comm., 180, pp. 1730-1745, (2009).

[9] B. Després, Uniform asymptotic stability of Strang's explicit schemes, SIAM J. Numer. Anal. 47, pp. 3956-3976, (2009).

[10] E. Fijalkow, A numerical solution to the Vlasov equation, Comput. Phys. Commun. 116, pp. 319-328, (1999).

[11] F. Filbet, E. Sonnendrücker, Comparison of Eulerian Vlasov solvers, Comput. Phys. Commun. 151, pp. 247-266, (2003).

[12] H.E. Heath, I.M. Gamba, P.J. Morrison, C. Michler, A discontinuous Galerkin method for the Vlasov-Poisson system, preprint.

[13] A. Mangeney, F. Califano, C. Cavazzoni, P. Travnicek, A Numerical Scheme for the Integration of the VlasovMaxwell System of Equations, J. Comput. Phys. 179, 495538 (2002).

[14] M. Mehrenberger, Splitting d'ordre 4 pour Vlasov-Poisson, in SMAI congress 2011, Guidel, http://smai.emath.fr/smai2011/ slides/mehrenbe/Slides.pdf.

[15] K.W. MonTon, Shock capturing, fitting and recovery, Proceeding 8-th Internat. Conf. Numer. Meth. in Fluid Dynamics, Lecture note in Physics 170, E. Krause Ed., Springer-Verlag, Berlin, 1982, pp. 77-93

[16] K.W. Monton, Generalized Galerkin methods for hyperbolic problems, Comput. Methods Appl. Mech. Engrg. 52, pp. 847-871, 1985.

[17] K.W. Morton, A. Priestley, E. Suli, Stability of the Lagrange-Galerkin method with non-exact integration, RAIRO 22, pp. 625-653, (1988).

[18] T. Nakamura, T. YaBe, Cubic interpolated propagation scheme for solving the hyper-dimensional Vlasov-Poisson equation in phase space, Comput. Phys. Commun. 120, pp. 122-154, (1999).

[19] J. QIU, Positivity preserving semi-Lagrangian discontinuous Galerkin method and application to kinetic equations, Joint SIAM/RSME-SCM-SEMA Meeting, June 2010. 
[20] J.A. Rossmanith, D.C. Seal, A positivity-preserving high-order semi-Lagrangian discontinuous Galerkin scheme for the Vlasov-Poisson equations, J. Comp. Phys. 230, pp. 6203-6232, (2011).

[21] E. SonnendrüCKer, Approximations numérique des équations Vlasov-Maxwell, (Notes du Cours M2), (2010).

[22] M. Shoucri, Nonlinear evolution of the bump-on-tail instability, Phys. Fluids 22, p. 38, (1978).

[23] G. Strang, On the construction and comparison of difference schemes, SIAM J. Numer. Anal. 5, pp. 506-517, (1968).

[24] T.H. Watanabe, H. Sugama, Vlasov and drift-kinetic simulation methods based on symplectic integrators, Transp. Theory and Stat. Phys., 34, pp. 287-309, (2005).

[25] M. Zhang, C.-W. Shu, Fourier analysis for discontinuous Galerkin and related methods, Chinese Sci Bull, 2009, 54: 18091816, doi: $10.1007 /$ s11434-009-0365-2.

[26] X. Zhang And C.-W. Shu, On positivity preserving high order discontinuous Galerkin schemes for compressible Euler equations on rectangular meshes, J. Comput. Phys., 229 (2010), pp. 8918-8934. 